\section{ECCOMAS}

Proceedia
COMPDYN 2021

$8^{\text {th }}$ ECCOMAS Thematic Conference on Computational Methods in Structural Dynamics and Earthquake Engineering

M. Papadrakakis, M. Fragiadakis (eds.)

\title{
A NEURAL NETWORK-BASED APPROACH FOR SEISMIC-INDUCED DAMAGE ASSESSMENT OF STEEL LIQUID STORAGE TANKS
}

\author{
Laura Micheli $^{1}$ and Mahmoud Faytarouni ${ }^{2}$ \\ ${ }^{1}$ Dept. of Civil and Environmental Engineering, Catholic University of America \\ Washington, DC, USA \\ e-mail: michelil@cua.edu \\ ${ }^{2}$ Genex Systems, Turner Fairbank Highway Research Center \\ McLean, VA, USA
}

\begin{abstract}
Surrogate models, including neural network (NN), machine learning, and Kriging, are used in various fields to reduce the computational demand of risk assessment and uncertainty analysis. In civil engineering applications, surrogate models are usually trained on synthetic data generated with numerical simulation models, which might yield approximate responses and significant computational burdens. Post-disaster reconnaissance observations represent an alternative source of data that could be used to train a surrogate model without the need for numerical models. However, the limited number of reconnaissance observations available in the literature might yield challenges, such as unbalanced data distributions. This paper presents a surrogate-based approach for seismic-induced damage prediction, where postearthquake reconnaissance data are exploited to train a NN model. The approach is demonstrated on steel liquid storage tanks. Field data from past earthquake reconnaissance reports are first collected. Then, features representative of tank characteristics, seismic hazard parameters, and seismic-induced damage are extracted. A traditional two-layers $N N$ model is built to map the relationship between tank characteristics, seismic hazard parameters, and seismic-induced damage. To tackle the challenge of unbalanced dataset, a cascade NN approach is proposed. In the cascade approach, two NN models are employed to predict the damage level of the steel tanks. The first NN returns a binary classification of the tank damage (i.e., damage, no damage). If the tank results damaged, a second $N N$ identifies the level of damage (i.e., minor, severe, collapse). The performance of traditional and cascade NN approaches is compared using different metrics. Results demonstrate that the cascade NN strategy leads to more accurate damage predictions than the traditional NN approach.
\end{abstract}

Keywords: neural network, reconnaissance data, damage classification, steel tanks, surrogate model, seismic risk-assessment 


\section{INTRODUCTION}

Performance-Based Design (PBD) is gaining prominence in the civil engineering community [1]. PBD allows to holistically evaluate a structure for potential risks and engage building owners in cost-effective risk-mitigation strategies [2-4]. It follows that risk assessment is a key component of $\mathrm{PBD}$, as it enables the estimation of the probability of occurrence of a certain damage state, considering a variety of hazard scenarios [1,5]. Typically, the performance of civil structures is assessed through nonlinear numerical models (e.g., finite element models), which permit the evaluation of the structure's response beyond the elastic regime $[2,6,7]$. However, the computational demand of high-fidelity numerical simulation models, coupled with the necessity to simulate the structure under a large number of hazard and uncertainty scenarios, makes risk assessment a computationally demanding, if not impractical task.

Data-driven surrogate models [8-10], including neural network (NN), Kriging, and machine learning, have been proposed to reduce the computational burden of risk assessment $[11,12]$. Surrogate models, or metamodels, are simplified mathematical relationships trained based on input/output observations to represent the physical system under consideration. In civil engineering applications, metamodels have been used to replace computationally expensive numerical models for uncertainty and risk analyses. For example, Sudret and Mai [13] employed polynomial chaos expansion to generate fragility curves of a 3-story structure subjected to seismic hazard. Later, Gidaris et al. [14] developed a Kriging surrogate model for seismic risk assessment of civil structures. The authors exploited the metamodel to construct fragility functions of a 4-story building under stochastic ground motions and uncertain structural parameters. Recently, Micheli et al. [15] proposed a multiple-surrogate models framework for risk assessment of wind-excited tall buildings. The proposed framework exploits a set of Kriging surrogates to reduce the computational demand of risk assessment of high-rise structures subjected to wind load time histories. A drawback of using surrogate models for civil structures is the necessity to generate input/output observations to train the metamodel. Synthetic input/output observations are usually derived from numerical simulation models, which might lead to approximate responses. Furthermore, when the response of the structure is highly nonlinear, a large data pool might be required to train a reliable surrogate model [1517], yielding a significant computational burden. A solution to this drawback could be to extract input/output observations directly from field data [18]. In this context, post-disaster field reconnaissance reports could be a valuable source of information, enabling a realistic representation of damage mechanisms and hazard characteristics. However, the use of post-disaster reconnaissance observations presents some challenges. For example, the limited number of records available could yield unbalanced datasets, eventually reducing the accuracy of the surrogate model.

This paper investigates a surrogate-based approach for damage assessment of civil structures, where the metamodel is trained based on reconnaissance observations. The approach is demonstrated on aboveground, cylindrical, steel liquid storage tanks subjected to seismic hazard. The seismic risk of storage tanks is significantly higher than ordinary structures, as damage to liquid storage tanks could cause irreparable consequences to built and natural environments, including the release of hazardous materials, uncontrolled fires, and soil contaminations [19-21]. In the proposed approach, features representative of tank characteristics (e.g., diameter, height, thickness, liquid level) and seismic excitation parameters (e.g., peak ground acceleration, magnitude) are extracted directly from reconnaissance reports, along with the corresponding damage levels. Then, a surrogate model consisting of a two-layers NN is built to map the relationship between inputs (i.e., tank and hazard features) and output (i.e., damage level). To tackle the unbalanced dataset challenge, a cascade approach is proposed, 
where two NN models are employed to estimate the damage level of steel tanks. The first NN exploits a binary classification to guess if the tank is damaged or not. If the tank results damaged, a second $\mathrm{NN}$ identifies the damage level between minor damage, severe damage, and collapse. The accuracy of the NNs is assessed on a testing dataset using different performance metrics, such as accuracy, precision, and recall. The performance at predicting seismicinduced damage of the traditional two-layers NN and the cascade NN models is compared.

The remainder of the paper is organized as follows. First, the collected post-earthquake reconnaissance data are described. Then, traditional and cascade $\mathrm{NN}$ approaches are introduced. After, the metrics employed to quantify the performance of the NNs are discussed. Finally, the damage prediction capability of traditional and cascade NNs is compared.

\section{METHODOLOGY}

\subsection{Dataset Description and Features Extraction}

In this study, post-earthquake reconnaissance observations are employed as data pool for training a NN model. Such observations were derived from existing post-earthquake reconnaissance report [22] and survey [23]. Both anchored and unanchored cylindrical steel tanks were taken in consideration. Features representative of tank and seismic hazard properties were extracted and used as inputs of the NN models described in Section 2.2. Tank features include geometric characteristics, such as diameter $(D)$, height $(H)$, shell thickness $(t)$, and height of the liquid at the moment of the earthquake $\left(H_{L}\right)$. Peak ground acceleration (PGA) and magnitude $(M)$ were selected as seismic hazard features. For some of the tanks, the thickness was not documented in the post-earthquake report in reference [22]. The thickness of these tanks was estimated based on the work of Cortes and Prinz [24]. Similarly, for some earthquake events, the PGA was not reported, and it was estimated based on the location of the tank and the corresponding ground motion records [25]. The material properties of the steel tanks were not enclosed in the reports. Therefore, yielding stress and modulus of elasticity of steel were assumed to be the same for all tanks and were not included between the input variables. A total of $n=247$ aboveground, cylindrical, steel liquid storage tanks observations were collected. Table 1 reports the statistics of the dataset, while Figure 1 (a) shows a schematic representation of a storage tank, including the features selected to represent tank and seismic hazard characteristics.

\begin{tabular}{lllll}
\hline & Min. & Max. & Mean & Standard dev. \\
\hline Diameter, $D(\mathrm{~m})$ & 2.0 & 83 & 22 & 14.3 \\
Height, $H(\mathrm{~m})$ & 3.0 & 45 & 11 & 4.0 \\
Thickness, $t(\mathrm{~m})$ & 0.001 & 0.098 & 0.01 & 0.01 \\
Liquid height, $H_{L}(\mathrm{~m})$ & 0.0 & 44 & 10 & 8.4 \\
Magnitude, $M$ & 5.5 & 8.4 & 7.0 & 0.64 \\
Peak ground acc., PGA $(\mathrm{g})$ & 0.02 & 2.0 & 0.41 & 0.40 \\
\hline
\end{tabular}

Table 1: Statistics of the dataset.

A description of the damage reported by each tank was also retrieved from the reconnaissance reports [20,21], along with the corresponding values of tank and seismic features. The 
steel tanks experienced various types of seismic-induced damage, including elephant foot buckling, roof damage, shell buckling, and complete collapse [20,21]. The damage types were assembled in four main classes: class 0, no damage; class 1, minor damage; class 2, severe damage; and class 3, collapse. Minor damage includes damage to the seal, minor roof damage, damaged valves, and, in general, damage that requires simple repairs. Severe damage comprises elephant foot buckling, shell buckling, local buckling caused by roof collapse, and floor plate rupture. Collapse is intended as the tank's total failure, or widely spread damage that necessitates the replacement of the tank. Note that, if more reconnaissance observations become available, additional damage mechanisms could be included and the damage classes may be further refined. Figure 1 (b) shows the distribution of the steel tank observations among the four damage classes, where $n_{0}$ represents the number of data in class $0, n_{1}$ in class $1, n_{2}$ in class 2 , and $n_{3}$ in class 3 , respectively. Figure 1 demonstrates that the dataset is severely skewed toward one class only, with the majority of data lying in class 0 , and a smaller number of observations belonging to class 3. Such an unbalanced dataset could cause poor performance of traditional $\mathrm{NN}$ models, as the majority of the $\mathrm{NN}$ algorithms available in the literature were developed considering a balanced data distribution [26].



(a)

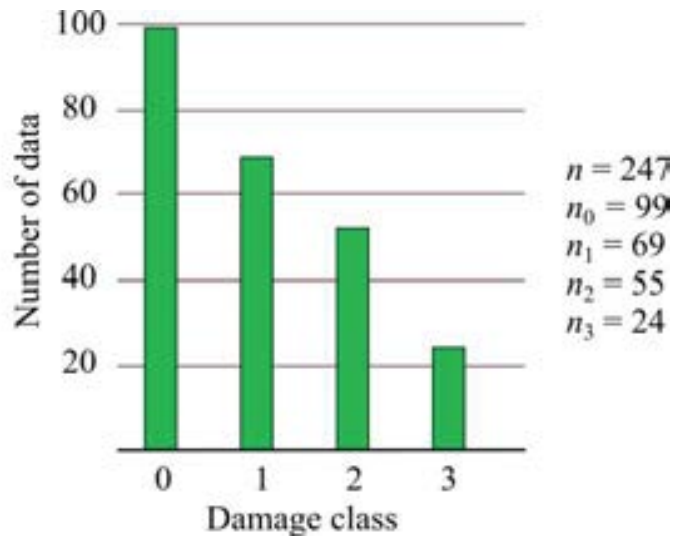

(b)

Figure 1: (a) Schematic representation of an aboveground, cylindrical, steel liquid storage tank and input variables; (b) distribution of the data among the four damage classes.

\subsection{Neural Network for Damage Prediction}

A traditional two-layers NN is first used to map the relationship between tank and seismic hazard characteristics (i.e., inputs) and the corresponding damage class (i.e., output). The NN is composed of a hidden and an output layer, with sigmoid and softmax activation functions, respectively. Figure 2 illustrates a representation of the $\mathrm{NN}$ architecture. In the figure, $k_{1}$ and $k_{2}$ indicate the number of neurons in the hidden and output layers, respectively. The NN has six inputs, corresponding to tank and seismic hazard features, and one output, representing the damage class. The output of the NN can be expressed as [27]:

$$
y=g\left(f\left(\mathbf{w}_{1} \mathbf{x}+\mathbf{b}_{1}\right)\right) \mathbf{w}_{2}+\mathbf{b}_{2}
$$

where $\mathbf{x}$ is the input vector, $\mathbf{w}_{1}$ and $\mathbf{w}_{2}$ are the hidden and the output layers' weights, $\mathbf{b}_{1}$ and $\mathbf{b}_{2}$ are the hidden and the output layers' biases, $f$ and $g$ are the sigmoid and softmax activation 
functions, respectively. The weights and biases are tuned based on the training dataset using a gradient backpropagation algorithm [27].

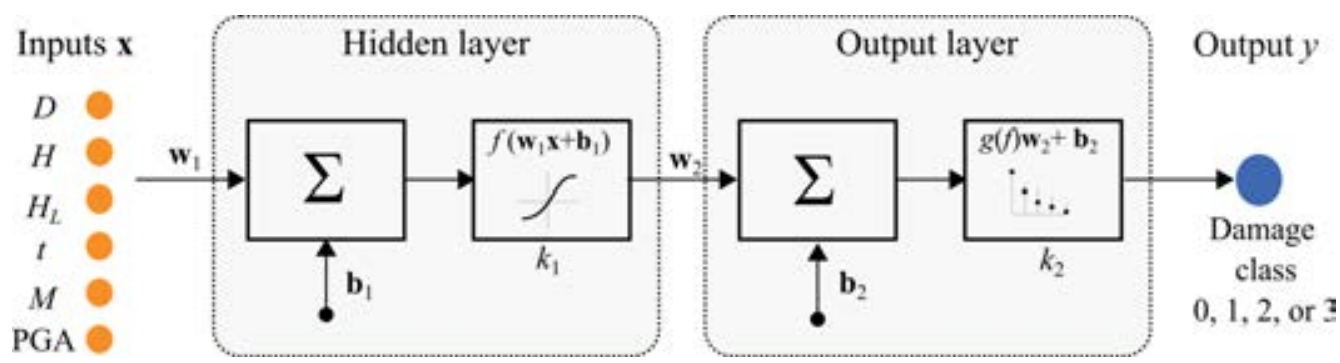

Figure 2: Neural Network configuration.

To tackle the unbalanced dataset issue (Figure 1 (b)), a cascade NN approach is proposed as an alternative for seismic-induced damage prediction. Instead of a single $\mathrm{NN}$, the cascade approach employs two consecutive NN algorithms to estimate the damage class experienced by a steel tank as a function of the input variables. The first NN is trained based on a binary classification of the data among class 0 (no damage) and class 1 (damage). Note that in the binary classification, all the damaged tanks are lumped in class 1, independently of the type of damage they experienced. A second $\mathrm{NN}$ classifies the type of damage among classes 1,2 , and 3 , eliminating the data in class 0 , and thus, the source of unbalance. Both $\mathrm{NN}$ models have the same inputs (tank and seismic hazard features), but different outputs and number of training data. The proposed approach is schematically represented in Figure 3 (a).

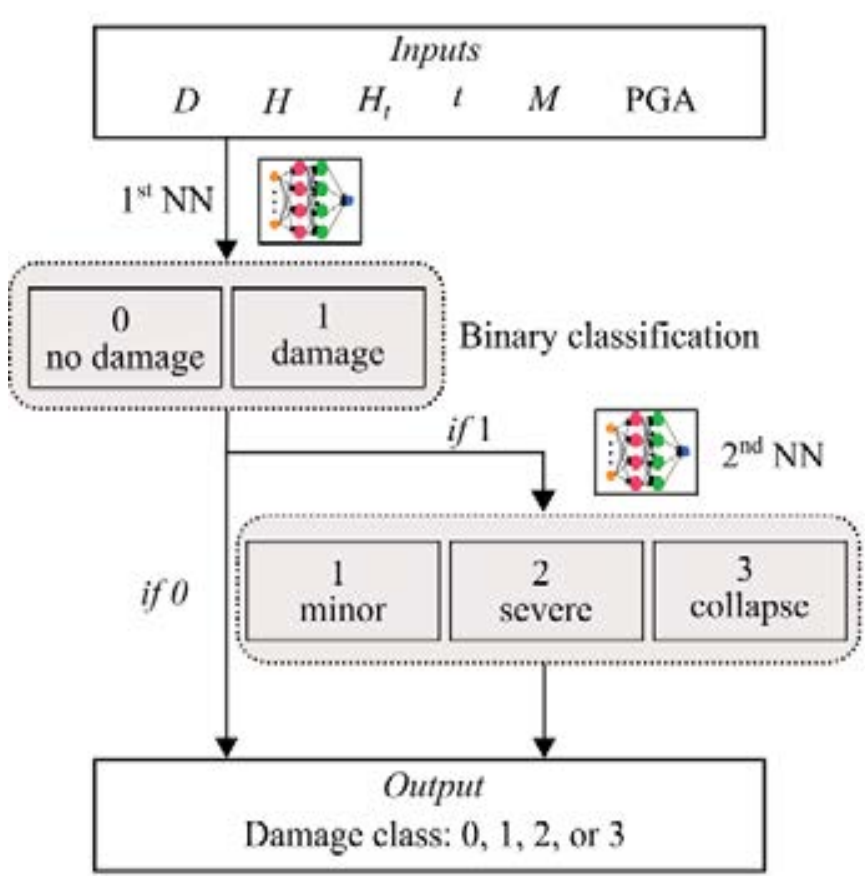

(a)

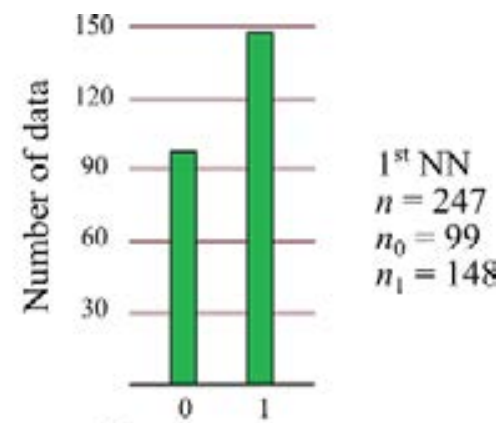

Damage class

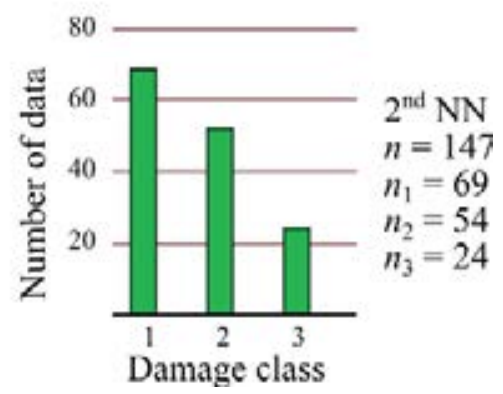

(b)

Figure 3: (a) Cascade neural network representation; (b) data distribution among damage classes for the $1^{\text {st }}$ and the $2^{\text {nd }} \mathrm{NN}$ models in the cascade approach. 
It is worth noting that the cascade approach uses the dataset described in Section 2.1, where data were first rearranged in the binary classification damage/no damage. Then, for training the second $\mathrm{NN}$, data belonging to class 0 were eliminated, leaving only observations lying in classes 1,2 , and 3 . The distribution of the data among different damage classes is reported in Figure 3 (b) for the two NN models. Furthermore, the architecture of the two NNs of the cascade approach is the same as the one shown in Figure 2.

\subsection{Performance Metrics}

The effectiveness of both traditional NN and cascade NNs approaches at predicting the damage experienced by steel liquid storage tanks subjected to seismic hazard is assessed on a testing subset. Specifically, the tank dataset composed of $n$ observations is divided into three subsets: training, validation, and testing. The training data (size $n_{t r}=70 \% n$ ) is used to train the NN models. The validation subset (size $n_{v a l}=15 \% n$ ) is employed to validate the models, while the testing subset ( size $n_{t s}=15 \% n$ ) is exploited to test the prediction capability of the NNs. The performance of the NNs at predicting the damage class in correspondence with the testing data is taken as an indication of the model's performance on unseen data.

\begin{tabular}{llllll}
\hline & & $n$ & $n_{\text {tr }}$ & $n_{\text {val }}$ & $n_{\text {ts }}$ \\
\hline Traditional & $\mathrm{NN}$ & 247 & 173 & 37 & 37 \\
\multirow{2}{*}{ Cascade } & $1^{\text {st }} \mathrm{NN}$ & 247 & 173 & 37 & 37 \\
& $2^{\text {nd }} \mathrm{NN}$ & 147 & 103 & 22 & 22 \\
\hline
\end{tabular}

Table 2: Dataset sizes for the traditional NN and cascade NN models.

The performance of the NNs is evaluated using different metrics, including precision, recall, and accuracy. The precision can be defined as the percentage of damage cases correctly assigned by the NN. The recall represents the percentage of the true damage classes correctly predicted by the algorithm, also referred to as true positives. The accuracy is defined as the ratio between the number of correct predictions and the total number of observations. Precision and recall can be computed for each class, while accuracy is a global performance metric.

\section{RESULTS AND DISCUSSION}

\subsection{Neural Network}

The two-layer NN model described in Figure 2 was trained to predict the damage class experienced by the tanks between 0 (no damage), 1 (minor damage), 2 (severe damage), and 3 (collapse) with the dataset described in Section 2.1 and reported in Figure 1(b). MATLAB Toolbox [28] was used to build, train, and test all the NN models developed in this study. The number of neurons $k_{1}$ and $k_{2}$ were selected iteratively to maximize the accuracy on the validation subset and set as $k_{1}=40$ and $k_{2}=3$. The values of weights and biases obtained from the training process can be found in reference [29]. Note that the trained NN model can be employed to predict the damage level of steel tanks with characteristics lying in the ranges reported in Table 1 only.

Precision, recall, and accuracy for training, validation, and testing datasets are reported in the confusion matrices in Figure 4. Each element of the confusion matrix $C_{i, j}$ indicates the 
number of data known to be in class $i$ and predicted in class $j$. The diagonal elements in green are the damage classes correctly guessed by the NN model, while the off-diagonal elements in red are the categories predicted incorrectly. The confusion matrices also contain precision and recall for each class, along with overall accuracy of the model.



(a)

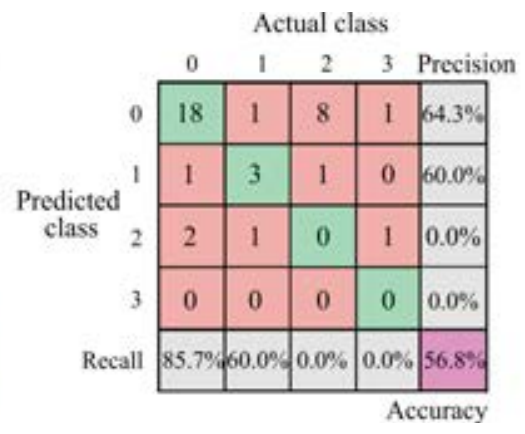

(b)

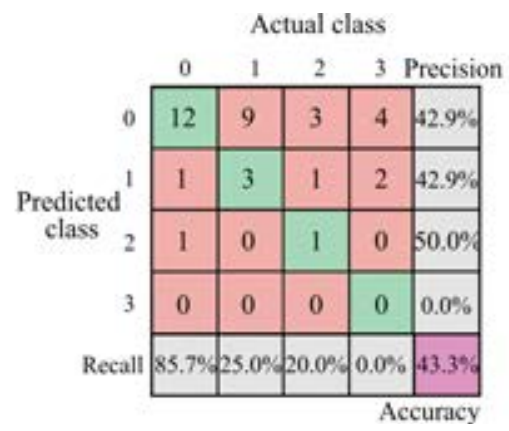

(c)

Figure 4: Confusion matrix for the traditional NN: (a) training, (b) validation, and (c) testing datasets.

Results in Figure 4 indicate a relatively poor performance of the $\mathrm{NN}$ at predicting the damage classes, with an average accuracy of $47.6 \%$ (accuracy averaged over training, validation, and testing datasets). Looking at recall and precision, it can be observed that the simplest class to predict is class 0 , where the majority of the data is concentrated. The lowest values of precision and recall correspond to class 3 , which corresponds to the class with the smallest number of observations available (Figure 1(b)). The poor performance of the NN can be attributed to the unbalance in the distribution of the data, which are severely skewed toward class 0 . In particular, Figure 4 (a) shows that a very small number of data were used to train the NN in class 3, which might translate to poor accuracy of the model over both validation and testing datasets, as demonstrated by Figure 4 (b) and (c).

\subsection{Cascade Neural Networks}

In the cascade approach, two NN models were built and employed to classify the damage level of steel tanks, following the procedure summarized in Figure 3 (a). The first NN model was trained to predict the binary damage classification 0 (no damage) and 1 (damage) based on the data distribution reported in Figure 3 (b). The second NN was trained to identify the damage level of the tank between class 1 (minor damage), class 2 (severe damage), and class 3 (collapse). For both NN models, the number of neurons was selected iteratively as $k_{1}=10$ and $k_{2}=3$, while weights and biases values can be found in reference [29]. Figure 5 illustrates the confusion matrices for the first NN, while Figure 6 reports the second NN results.

Results in Figures 5 and 6 show that both NN models yield similar performance, with an average accuracy of $73.4 \%$ for the first $\mathrm{NN}$ and $71 \%$ for the second model. For the first NN, the highest values of precision are reached in class 1 , while the largest recall correspond to class 0 in training and validation datasets, and class 1 in the testing subset. On the other hand, results in Figure 6 show that class 1 seems to be the simplest class to predict with the second $\mathrm{NN}$ model, corresponding to relatively high values of precision and recall over training, validation, and testing datasets.

A cross-comparison between the performance of the traditional and the cascade NN approaches demonstrate that the cascade NNs are $51.7 \%$ more accurate than the single NN. This 
could be attributed to the more balanced distribution of the observations among different damage classes in the cascade approach. Overall, the performance of the models in terms of recall and precision varies with the damage class under consideration, with the highest values attained by the two cascade NN models. Based on the overall accuracy and the fair performance at identifying different damage classes, the cascade NN approach is recommended as the method to use to estimate the damage level of steel storage tanks as a function of tank and seismic hazard characteristics.

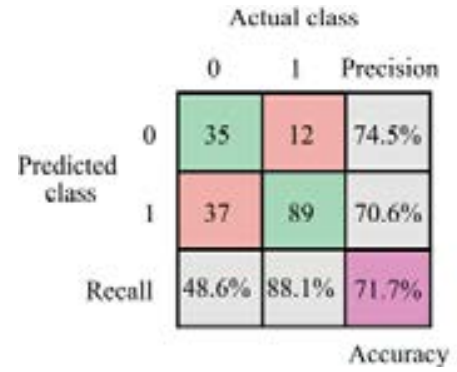

(a)

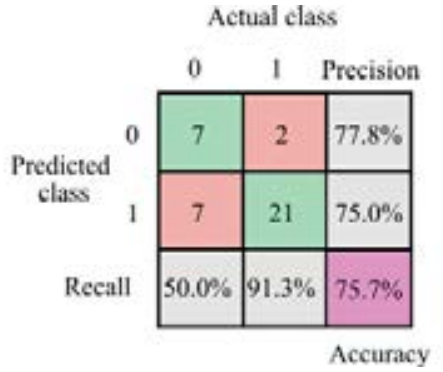

(b)

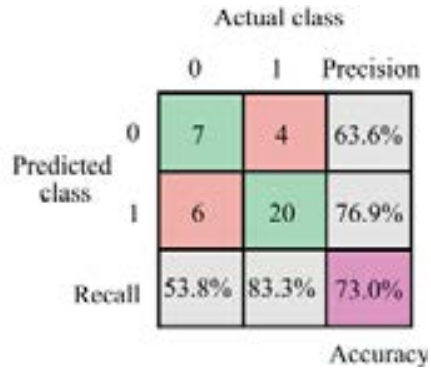

(c)

Figure 5: Confusion matrix for the $1^{\text {st }} \mathrm{NN}$ of the cascade approach: (a) training, (b) validation, and (c) testing datasets.

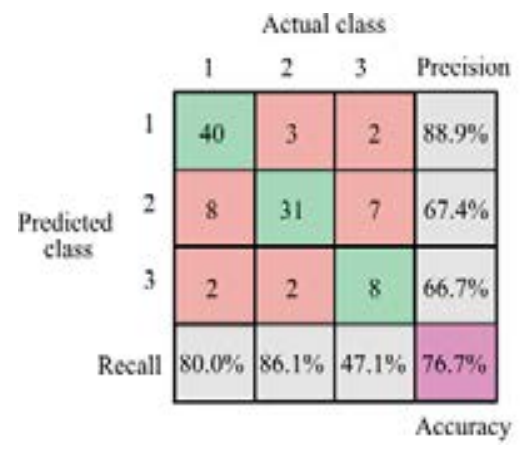

(a)

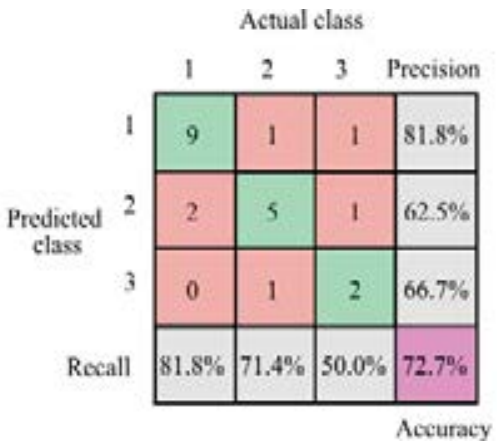

(b)

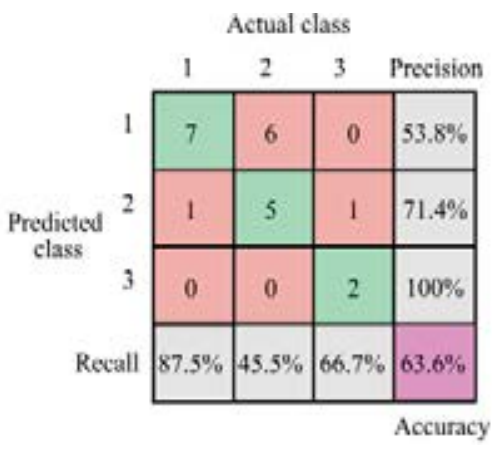

(c)

Figure 6: Confusion matrix for the $2^{\text {nd }} \mathrm{NN}$ of the cascade approach: (a) training, (b) validation, and (c) testing datasets.

\section{CONCLUSIONS}

In this paper, an NN-based approach for damage prediction of aboveground, cylindrical, steel liquid storage tanks was investigated. In the proposed method, data were derived from post-earthquake reconnaissance reports and surveys. Features representative of tank and seismic hazard characteristics were extracted from the reconnaissance reports, along with a description of the corresponding damage modes. The different types of damage were initially classified into four classes, ranging from no damage to collapse. The limited number of reconnaissance observations available in the literature yielded an unbalanced dataset, with the majority of the data skewed toward one class. First, a traditional two-layers NN was constructed to infer the unknown relationship between tank and seismic features (inputs of the 
NN model), and corresponding damage class (output). Then, to tackle the unbalanced dataset challenge, a cascade NN approach was proposed, where two NN models were utilized to guess the damage class of the tanks. The first NN was trained based on a binary classification of the reconnaissance observations (i.e., damage, no damage). If the tank resulted in the damage class from the first model, a second $\mathrm{NN}$ refined the damage guess between minor damage, severe damage, and collapse. The performance of traditional and cascade NN methods was assessed with different metrics, such as accuracy, precision, and recall.

Results demonstrated that the cascade NN approach led to a better performance than the conventional NN model, with an average accuracy of $73.4 \%$ for the first and $71 \%$ for the second NN models. On the other hand, the traditional NN yielded a relatively poor performance, with an average accuracy of $47.6 \%$. The values of recall and precision vary with the damage class under consideration. Overall, the two NNs in the cascade approach resulted in the largest values of both precision and recall compared to the traditional model. The enhanced performance of the cascade NNs can be attributed to the more balanced distribution of the data relatively to the traditional $\mathrm{NN}$ dataset.

Overall, the paper showed that the cascade $\mathrm{NN}$ approach could be a viable alternative to traditional NN models, in particular when unbalanced datasets are available, such as for postdisaster reconnaissance observations. Nevertheless, the accuracy of the cascade NN models could to be further improved by increasing the number of training data or using different data learning algorithms. Future investigations will entail exploring different machine learning algorithms, applying other techniques to solve the issue of imbalanced data, and integrating the cascade NNs into a seismic risk assessment framework for steel liquid storage tanks.

\section{ACKNOWLEDGEMENTS}

This study was supported by Catholic University of America's 2020 Burns Junior Faculty Fellowship. Any opinions, finding, and conclusion or recommendation expressed in this material are those of the authors and do not necessarily reflects the view of the sponsors.

\section{REFERENCES}

[1] Federal Emergency Management Agency (FEMA), "Seismic performance assessment of buildings, Volume 1 - Methodology," FEMA Pubblication P-58-1, Washington, DC, 2012.

[2] Yang, T. Y., Moehle, J., Stojadinovic, B., \& Der Kiureghian, A., "Seismic performance evaluation of facilities: methodology and implementation," Journal of Structural Engineering, pp. 135(10), 1146-1154, 2009.

[3] Ierimonti, L., Caracoglia, L., Venanzi, I., \& Materazzi, A. L., "Investigation on life-cycle damage cost of wind-excited tall buildings considering directionality effects," Journal of Wind Engineering and Industrial Aerodynamics, pp. 171, 207-218, 2017.

[4] Micheli, L., Alipour, A., Laflamme, S., \& Sarkar, P., "Performance-Based Design with Life-Cycle Cost Assessment for Damping Systems Integrated in Wind Excited Tall Buildings," Engineering Structures, pp. 195, 438-451, 2019 b.

[5] Faytarouni, M., Shen, J., Seker, O., \& Akbas, B, "Seismic demand on column splices in concentrically braced frames considering brace fracture," Journal of Constructional Steel Research, pp. 175, 106350, 2020.

[6] Faytarouni, M., Seker, O., Akbas, B., \& Shen, J. , "Impact of Brace Fracture on Column Splice Demands in Braced Frames," Journal of Structural Engineering, pp. 146(8), 
04020152, 2020.

[7] Caprili, S., Panzera, I., \& Salvatore, W., "Resilience-based methodologies for design of steel structures equipped with dissipative devices," Engineering Structures, vol. 228, p. $111539,2021$.

[8] Sun, H., Burton, H., \& Wallace, J. , "Reconstructing seismic response demands across multiple tall buildings using kernel-based machine learning methods," Structural Control and Health Monitoring, vol. 26(7), p. e2359, 2019.

[9] Kiani, J., Camp, C., \& Pezeshk, S. , "On the application of machine learning techniques to derive seismic fragility curves," Computers \& Structures, vol. 218, pp. 108-122, 2019.

[10] Sun, H., Burton, H. V., \& Huang, H. , "Machine learning applications for building structural design and performance assessment: state-of-the-art review," Journal of Building Engineering, p. 101816, 2020.

[11] Moustapha, M., Bourinet, J. M., Guillaume, B., \& Sudret, B., "Comparative Study of Kriging and Support Vector Regression for Structural Engineering Applications," ASCEASME Journal of Risk and Uncertainty in Engineering Systems, Part A: Civil Engineering, pp. 4(2), 04018005, 2018.

[12] Abdallah, I., Lataniotis, C., \& Sudret, B., "Parametric hierarchical kriging for multifidelity aero-servo-elastic simulators-Application to extreme loads on wind turbines," Probabilistic Engineering Mechanics, pp. 55, 67-77, 2019.

[13] Sudret, B., \& Mai, C. V., "Computing seismic fragility curves using polynomial chaos expansions," in 11th International Conference on Structural Safety and Reliability (ICOSSAR), Zürich, 2013.

[14] Gidaris, I., Taflanidis, A. A., \& Mavroeidis, G. P., "Kriging metamodeling in seismic risk assessment based on stochastic ground motion models," Earthquake Engineering \& Structural Dynamics, pp. 44(14), 2377-2399, 2015.

[15] Micheli, L., Alipour, A., \& Laflamme, S. , "Multiple-Surrogate Models for Probabilistic Performance Assessment of Wind-Excited Tall Buildings under Uncertainties," ASCEASME Journal of Risk and Uncertainty in Engineering Systems, Part A: Civil Engineering, vol. 6(4), p. 04020042, 2020.

[16] Le, V., \& Caracoglia, L. , "A neural network surrogate model for the performance assessment of a vertical structure subjected to non-stationary, tornadic wind loads," Computers \& Structures, vol. 231, p. 106208, 2020.

[17] Micheli, L., \& Laflamme, S, "Kriging-based design for robust high-performance control systems," ASCE-ASME Journal of Risk and Uncertainty in Engineering Systems, Part A: Civil Engineering, pp. 6(4), 04020037, 2020.

[18] Mangalathu, S., Sun, H., Nweke, C. C., Yi, Z., \& Burton, H. V. , "Classifying earthquake damage to buildings using machine learning," Earthquake Spectra, vol. 36(1), pp. 183208, 2020.

[19] Bakalis, K., Kazantzi, A. K., Vamvatsikos, D., \& Fragiadakis, M, "Seismic performance evaluation of liquid storage tanks using nonlinear static procedures," Journal of Pressure Vessel Technology, p. 141(1), 2019.

[20] Bakalis, K., Fragiadakis, M., \& Vamvatsikos, D. , "Surrogate modeling for the seismic performance assessment of liquid storage tanks," Journal of Structural Engineering, vol. 143(4), p. 04016199, 2017.

[21] Bohra, H., Azzuni, E., \& Guzey, S. , "Seismic analysis of open-top storage tanks with 
flexible foundation," Journal of Pressure Vessel Technology, vol. 141(4), 2019.

[22] Cooper, T. W, "A study of the performance of petroleum storage tanks during earthquakes, 1933-1995," US National Institute of Standards and Technology, 1997.

[23] Hamdan, F. H, "Seismic behaviour of cylindrical steel liquid storage tanks," Journal of Constructional steel research, pp. 53(3), 307-333., 2000.

[24] Cortes, G., \& Prinz, G. S, "Seismic fragility analysis of large unanchored steel tanks considering local instability and fatigue damage," Bulletin of Earthquake Engineering, pp. 15(3), 1279-1295, 2017.

[25] Pacific Earthquake Engineering Research Center, " PEER ground motion database," 2020. [Online]. Available: https://ngawest2.berkeley.edu/.

[26] Gu, Q., Cai, Z., Zhu, L., \& Huang, B. , "Data mining on imbalanced data sets," in In 2008 International Conference on Advanced Computer Theory and Engineering, (pp. 1020-1024). IEEE, 2008.

[27] Beale, M. H., Hagan, M. T., \& Demuth, H. B. , "Neural network toolbox. User's Guide," MathWorks, 2010.

[28] MathWorks, "MATLAB Machine Learning and Deep Learning Toolbox Release 2020b," MathWorks, Inc., Natick, Massachusetts, United States, 2021.

[29] Micheli, L., "GitHub," 2021. [Online]. Available: https://github.com/lauramch/COMPDYN2021. 